\title{
Active vibration control of piezolaminated stiffened plates
}

\author{
A. Mukherjee *, S.P. Joshi, A. Ganguli \\ Department of Civil Engineering, Indian Institute of Technology Bombay, Mumbai 400 076, India
}

\begin{abstract}
The aim of this work is to present active vibration control of stiffened plates. A stiffened plate finite element with piezoelectric effects is formulated. The characteristic feature of the stiffener is that it can have any shape in plan and need not pass through the nodal lines of the finite element mesh. The coupling between the direct and the converse piezoelectric effects is neglected for simplicity. A velocity feedback algorithm is employed in the active control. Numerical examples for vibration control of isotropic and orthotropic stiffened plates have been presented.
\end{abstract}

Keywords: Active vibration control; Stiffened plates; Piezoelectric sensors and actuators; Generalized stiffener formulation

\section{Introduction}

Active vibration control of stiffened structures is an important problem in various practical situations. In aircraft structures, the wings and fuselage consist of a skin with an array of stiffening ribs. Such structures are subjected to dynamic loads and their control is of paramount importance in safe and smooth functioning of the system. Bailey and Hubbard [1] proposed the use of distributed piezoelectric transducers in active vibration control of beams. Anderson and Hagood [2] discussed issues related to simultaneous sensing and actuation in structural control. Tzou [3] gave exhaustive information on vibration control of beams, plates and shells using piezoelectric materials. Gaudenzi et al. [4] demonstrated the aspects of vibration control of beams with collocated PZT piezopatches using velocity and position feedback systems. Lam and $\mathrm{Ng}$ [5] presented theoretical formulation for laminated plates using classical plate theory and Navier solutions. A negative force-cum-moment feedback algorithm is used in controlling the dynamic response of the plates. Chandrashekhara and Agarwal [6] presented a finite element approach to active vibration control of laminated composite plates using piezoelectric devices. Investigations on vibration control of plates have been carried out and their reference is available in the literature $[5,7]$. In practice, however, plates are often re- inforced by stiffeners. These stiffeners alter the dynamic characteristics of the plate to a great extent. Therefore, inclusion of the stiffeners in the model is very important. Moreover, the stiffeners vary widely in shape, size, orientation and number. In the existing literature very few publications on vibration control of stiffened plates are available. Some of these include piezoelectric stiffeners [7,8], stack actuators in angle stiffened plates [9] and layered plates [10].

In this paper, we present a general formulation for the active vibration control of stiffened plates with piezoelectric actuators. The stiffener can take any arbitrary shape in plan and the element can accommodate the stiffener anywhere in the finite element mesh. This feature liberates the finite element mesh from the rigors of accommodating the stiffeners of varying planforms along the nodal lines. The efficacy of the formulation has been demonstrated with several examples.

\section{Mathematical formulation}

The dynamic force equilibrium of a structure subjected to time-dependent forces can be written as a set of second-order differential equations in the following form:

$\mathbf{M} \ddot{\boldsymbol{\delta}}+\mathbf{C} \dot{\boldsymbol{\delta}}+\mathbf{K} \boldsymbol{\delta}=\mathbf{F}(\mathbf{t})$,

where $\mathbf{M}, \mathbf{C}$ and $\mathbf{K}$ are the mass, damping and stiffness matrices of the system and $\ddot{\boldsymbol{\delta}}, \dot{\boldsymbol{\delta}}$ and $\boldsymbol{\delta}$ are the acceleration, velocity and displacement vectors. The force vector $\mathbf{F}(\mathbf{t})$ comprises of the vectorial sum of external 
time-dependent forces and the control forces. In the following sections, we present the finite element formulation for the stiffened plate element. An eight-node plate element that can accommodate stiffeners of any shape in plan is formulated.

\subsection{Stiffness matrix}

The displacement field of a plate can be expressed as $U(x, y, z, t)=u_{0}-z \theta_{y}(x, y, t)$,

$V(x, y, z, t)=v_{0}+z \theta_{x}(x, y, t)$,

$W(x, y, z, t)=w_{0}(x, y, t)$,

where $U, V$ and $W$ are the displacements in $x, y$ and $z$ directions at any point in the element. The terms $u_{0}, v_{0}$ and $w_{0}$ are the reference plane displacements, and $\theta_{x}$ and $\theta_{y}$ are the rotations of transverse normal about $x$ and $y$ axes. The detailed formulation of the plate element is discussed elsewhere [11]. The stiffener formulation is elaborated in the present paper. For the stiffener all the parameters such as geometry as well as displacements are expressed in terms of the plate shape functions. Since the stiffener can be of any planform the displacement field at any point of the stiffener has been expressed in terms of the local co-ordinates tangential to the stiffener at that point. The displacement field is as follows (Fig. 1):

$\overline{\boldsymbol{\delta}}^{\prime}=\left\{\begin{array}{c}U^{\prime} \\ V^{\prime} \\ W^{\prime}\end{array}\right\}=\left\{\begin{array}{c}u_{0}^{\prime}-z \theta_{y^{\prime}} \\ z \theta_{x^{\prime}} \\ w_{0}+c \theta_{x^{\prime}}\end{array}\right\}$,

where the variables in primes depict the local co-ordinates.

The strain components for the stiffener element in $x^{\prime}-y^{\prime}$ system are given as

$$
\begin{aligned}
\boldsymbol{\epsilon}_{x^{\prime}} & =\left\{\begin{array}{l}
\varepsilon_{x} \\
\gamma_{x z} \\
\gamma_{x y}
\end{array}\right\}=\left\{\begin{array}{c}
\frac{\partial U^{\prime}}{\partial x^{\prime}} \\
\frac{\partial U^{\prime}}{\partial z}+\frac{\partial W^{\prime}}{\partial x^{\prime}} \\
\frac{\partial U^{\prime}}{\partial y^{\prime}}+\frac{\partial V^{\prime}}{\partial x^{\prime}}
\end{array}\right\} \\
& =\left\{\begin{array}{c}
\frac{\partial u_{0}^{\prime}}{\partial x^{\prime}}-z \frac{\partial \theta_{y^{\prime}}}{\partial x^{\prime}} \\
\frac{\partial w_{0}}{\partial x^{\prime}}-\theta_{y^{\prime}}+c \frac{\partial \theta_{x^{\prime}}}{\partial x^{\prime}} \\
z \frac{\partial \theta_{x^{\prime}}}{\partial x^{\prime}}
\end{array}\right\} .
\end{aligned}
$$

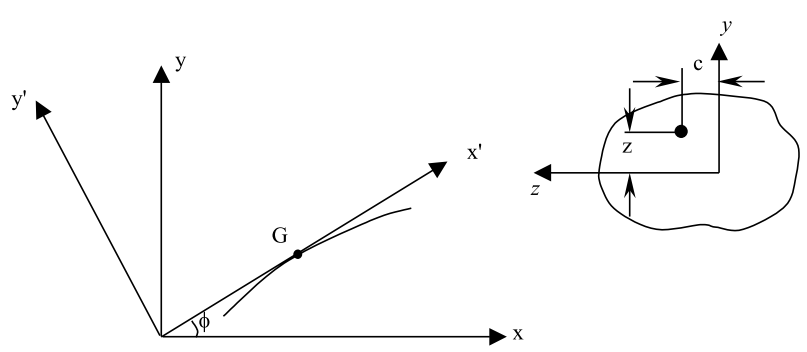

Fig. 1. A stiffener with its cross-section.
Eq. (3) is written in concise form as

$\boldsymbol{\epsilon}_{x^{\prime}}=\mathbf{H} \bar{\epsilon}_{x^{\prime}}$

where

$\mathbf{H}=\left[\begin{array}{rrrr}1 & -z & 0 & 0 \\ 0 & 0 & 1 & c \\ 0 & 0 & 0 & z\end{array}\right]$,

$\boldsymbol{\epsilon}_{x^{\prime}}$ are the strain components at any point in a crosssection of the stiffener, and $\overline{\boldsymbol{\epsilon}}_{x^{\prime}}$ are the strain components in terms of the displacements of the reference axis.

The relationship between the global $x$ co-ordinates and the local $x^{\prime}$ and $y^{\prime}$ co-ordinates is as follows:

$$
\begin{aligned}
& \left\{\begin{array}{l}
u_{0}^{\prime} \\
\theta_{x^{\prime}} \\
\theta_{y^{\prime}}
\end{array}\right\}=\left[\begin{array}{cccc}
\cos \phi & \sin \phi & 0 & 0 \\
0 & 0 & \cos \phi & \sin \phi \\
0 & 0 & -\sin \phi & \cos \phi
\end{array}\right]\left\{\begin{array}{l}
u_{0} \\
v_{0} \\
\theta_{x} \\
\theta_{y}
\end{array}\right\}, \\
& \left\{\begin{array}{l}
x \\
y
\end{array}\right\}=\left\{\begin{array}{l}
u_{0}^{\prime} \cos \phi-v_{0}^{\prime} \sin \phi \\
u_{0}^{\prime} \sin \phi+v_{0}^{\prime} \cos \phi
\end{array}\right\} .
\end{aligned}
$$

The derivatives of $x$ and $y$ with reference to $x^{\prime}$ are given by

$\frac{\partial x}{\partial x^{\prime}}=\cos \phi, \quad \frac{\partial y}{\partial x^{\prime}}=\sin \phi$.

The components of the generalized strain vectors are obtained as follows:

$$
\begin{aligned}
\frac{\partial u_{0}^{\prime}}{\partial x^{\prime}}= & \frac{\partial u_{0}^{\prime}}{\partial x} \frac{\partial x}{\partial x^{\prime}}+\frac{\partial u_{0}^{\prime}}{\partial y} \frac{\partial y}{\partial x^{\prime}} \\
= & \frac{\partial}{\partial x}\left(u_{0} \cos \phi+v_{0} \sin \phi\right) \frac{\partial x}{\partial x^{\prime}} \\
& +\frac{\partial}{\partial y}\left(u_{0} \cos \phi+v_{0} \sin \phi\right) \frac{\partial y}{\partial x^{\prime}} \\
= & \frac{\partial u_{0}}{\partial x} \cos ^{2} \phi+\frac{1}{2}\left(\frac{\partial v_{0}}{\partial x}+\frac{\partial u_{0}}{\partial y}\right) \sin 2 \phi+\frac{\partial v_{0}}{\partial y} \sin ^{2} \phi,
\end{aligned}
$$

$$
\begin{aligned}
\frac{\partial \theta_{y^{\prime}}}{\partial x^{\prime}}= & \left(\frac{\partial \theta_{y^{\prime}}}{\partial x} \frac{\partial x}{\partial x^{\prime}}+\frac{\partial \theta_{y^{\prime}}}{\partial y} \frac{\partial y}{\partial x^{\prime}}\right) \\
= & \frac{\partial}{\partial x}\left(-\theta_{x} \sin \phi+\theta_{y} \cos \phi\right) \cos \phi \\
& +\frac{\partial}{\partial y}\left(-\theta_{x} \sin \phi+\theta_{y} \cos \phi\right) \sin \phi \\
= & \frac{\partial \theta_{y}}{\partial x} \cos ^{2} \phi+\frac{1}{2}\left(-\frac{\partial \theta_{x}}{\partial x}+\frac{\partial \theta_{y}}{\partial y}\right) \sin 2 \phi-\frac{\partial \theta_{x}}{\partial y} \sin ^{2} \phi,
\end{aligned}
$$




$$
\begin{aligned}
\frac{\partial \theta_{x^{\prime}}}{\partial x^{\prime}}= & \frac{\partial \theta_{x^{\prime}}}{\partial x} \frac{\partial x}{\partial x^{\prime}}+\frac{\partial \theta_{x^{\prime}}}{\partial y} \frac{\partial y}{\partial x^{\prime}} \\
= & \frac{\partial}{\partial x}\left(\theta_{x} \cos \phi+\theta_{y} \sin \phi\right) \cos \phi \\
& +\frac{\partial}{\partial y}\left(\theta_{x} \cos \phi+\theta_{y} \sin \phi\right) \sin \phi \\
= & \frac{\partial \theta_{x}}{\partial x} \cos ^{2} \phi+\frac{1}{2}\left(\frac{\partial \theta_{y}}{\partial x}+\frac{\partial \theta_{x}}{\partial y}\right) \sin 2 \phi+\frac{\partial \theta_{y}}{\partial y} \sin ^{2} \phi
\end{aligned}
$$

$$
\begin{aligned}
\frac{\partial w_{0}}{\partial x^{\prime}}-\theta_{y^{\prime}}= & \frac{\partial w_{0}}{\partial x} \frac{\partial x}{\partial x^{\prime}}+\frac{\partial w_{0}}{\partial y} \frac{\partial y}{\partial x^{\prime}} \\
= & \frac{\partial w_{0}}{\partial x} \cos \phi+\frac{\partial w_{0}}{\partial y} \sin \phi \\
& -\left(-\theta_{x} \sin \phi+\theta_{y} \cos \phi\right) \\
= & \frac{\partial w_{0}}{\partial x} \cos \phi+\frac{\partial w_{0}}{\partial y} \sin \phi+\theta_{x} \sin \phi-\theta_{y} \cos \phi
\end{aligned}
$$

The generalized strain vectors are obtained in terms of the global strains through the following and substituting $\cos \phi=c, \sin \phi=s$ and $\sin 2 \phi=2 s$ :
Using the principle of minimum potential energy the stiffness matrix is obtained as

$\mathbf{K}_{s}=\int \mathbf{B}_{s}^{\mathrm{T}} \mathbf{D}_{s} \mathbf{B}_{s} \mathrm{~d} s$,

where

$\mathbf{D}_{s}=\iint\left(\mathbf{H}^{\mathrm{T}} \overline{\mathbf{D}} \mathbf{H}\right) \mathrm{d} y \mathrm{~d} z$,

where $\overline{\mathbf{D}}$ is the matrix of elastic constants.

\subsection{Consistent mass matrix}

The consistent mass matrices of the plate and the stiffener element are developed by assuming acceleration fields similar to the displacement fields as shown in Eqs. (2) and (3). The details of mass matrix formulation for the plate element are given elsewhere [13]. In this paper, only the stiffener mass matrix is derived. The acceleration field of the stiffener is as follows:

$\ddot{\boldsymbol{\delta}}=\left\{\begin{array}{c}\ddot{U} \\ \ddot{V} \\ \ddot{W}\end{array}\right\}=\left\{\begin{array}{c}\ddot{u}_{0}^{\prime}-z \ddot{\theta}_{y^{\prime}} \\ z \ddot{\theta}_{x^{\prime}} \\ \ddot{w}_{0}+c \ddot{\theta}_{x^{\prime}}\end{array}\right\}$.

The above equation can be expressed in terms of the reference plane accelerations as

$\left\{\begin{array}{c}\frac{\partial u_{0}^{\prime}}{\partial x^{\prime}} \\ \frac{\partial w_{0}}{\partial x^{\prime}}-\theta_{y^{\prime}} \\ -\frac{\partial \theta_{x^{\prime}}}{\partial x^{\prime}} \\ -\frac{\partial \theta_{y^{\prime}}}{\partial x^{\prime}}\end{array}\right\}=\left[\begin{array}{ccccccccc}c^{2} & s^{2} & \frac{1}{2}(2 s) & 0 & 0 & 0 & 0 & 0 & 0 \\ 0 & 0 & 0 & -s^{2} & c^{2} & -\frac{1}{2}(2 s) & \frac{1}{2}(2 s) & 0 & 0 \\ 0 & 0 & 0 & \frac{1}{2}(2 s) & -\frac{1}{2}(2 s) & c^{2} & s^{2} & 0 & 0 \\ 0 & 0 & 0 & 0 & 0 & 0 & 0 & s & c\end{array}\right]\left\{\begin{array}{c}\frac{\partial u_{0}}{\partial x} \\ \frac{\partial v_{0}}{\partial y} \\ \frac{\partial u_{0}}{\partial y}+\frac{\partial v_{0}}{\partial x} \\ -\frac{\partial \theta_{x}}{\partial y} \\ -\frac{\partial \theta_{y}}{\partial x} \\ -\frac{\partial \theta_{x}}{\partial x} \\ -\frac{\partial \theta_{y}}{\partial y} \\ \frac{\partial w_{0}}{\partial y}+\theta_{x} \\ \frac{\partial w_{0}}{\partial x}-\theta_{y}\end{array}\right\}$.

Eq. (9) is written in concise form as

$\overline{\boldsymbol{\epsilon}}_{x^{\prime}}=\mathbf{T} \epsilon_{x}$

where $\boldsymbol{\epsilon}_{x}$ is the strain vector corresponding to the plate, and $\mathbf{T}$ is the transformation matrix. Thus, the strain-displacement matrix for the stiffener is expressed in terms of the plate strain-displacement matrix as $\ddot{\boldsymbol{\delta}}=\left[\begin{array}{rrrr}1 & 0 & 0 & -z \\ 0 & 0 & z & 0 \\ 0 & 1 & c & 0\end{array}\right]\left\{\begin{array}{c}\ddot{u}_{0}^{\prime} \\ \ddot{w}_{0} \\ \ddot{\theta}_{x}^{\prime} \\ \ddot{\theta}_{y}^{\prime}\end{array}\right\}=\mathbf{G}_{s} \overline{\boldsymbol{\delta}}^{\prime}$,

where $\mathbf{G}_{s}$ is the matrix relating the stiffener acceleration field to the acceleration vector $\stackrel{\mathscr{\boldsymbol { \delta }}}{\prime}^{\prime}$ at the reference plane of the plate in $x^{\prime}-y^{\prime}$ system and contains the co-ordinates

$\mathbf{B}_{s}=\mathbf{T B}_{r}=\left[\begin{array}{cc}\frac{\partial N_{r}}{\partial x} c^{2}+\frac{1}{2} \frac{\partial N_{r}}{\partial y}(2 s) & \frac{\partial N_{r}}{\partial y} s^{2}+\frac{1}{2} \frac{\partial N_{r}}{\partial x}(2 s) \\ 0 & 0 \\ 0 & 0 \\ 0 & 0\end{array}\right.$

$$
\left.\begin{array}{ccc}
\cline { 2 - 3 } & 0 & 0 \\
0 & \frac{\partial N_{r}}{\partial y} s^{2}+\frac{1}{2} \frac{\partial N_{r}}{\partial x}(2 s) & -\frac{\partial N_{r}}{\partial x} c^{2}-\frac{1}{2} \frac{\partial N_{r}}{\partial y}(2 s) \\
0 & -\frac{\partial N_{r}}{\partial y}(2 s)-\frac{\partial N_{r}}{\partial x} c^{2} & \frac{1}{2} \frac{\partial N_{r}}{\partial x}(2 s)-\frac{\partial N_{r}}{\partial y} s^{2} \\
\frac{N_{r}}{\partial y} s+\frac{\partial N_{r}}{\partial x} c & N_{r} s & -N_{r} c
\end{array}\right] .
$$


$y^{\prime}$ and $z$. Expressing reference plane accelerations in global co-ordinates

$\overline{\check{\boldsymbol{\delta}}}^{\prime}=\mathbf{T} \overline{\tilde{\boldsymbol{\delta}}}$.

Using the isoparametric shape functions the accelerations at any point in the reference axis of the plate $\ddot{\boldsymbol{\delta}}$ is expressed in terms of nodal accelerations as

$\overline{\ddot{\boldsymbol{\delta}}}=\mathbf{N}_{\mathbf{r}} \mathbf{I}_{\mathbf{8}} \ddot{\boldsymbol{\delta}}_{r}, \quad r=1, \ldots, n$,

where $\mathbf{N}_{\mathbf{r}}$ is the shape function of the node $r, \mathbf{I}_{\mathbf{8}}$ is an identity matrix of eighth order, $\ddot{\boldsymbol{\delta}}_{r}$ are the nodal accelerations, and $n$ is the number of nodes in the element.

The consistent mass matrix is written as

$$
\begin{aligned}
\mathbf{M}_{s} & =\int_{V}(\mathbf{G T N})^{\mathrm{T}} \rho \mathbf{I}(\mathbf{G T N}) \mathrm{d} V \\
& =\int_{L}(\mathbf{T N})^{\mathrm{T}} \mathbf{m}(\mathbf{T N}) \mathrm{d} s,
\end{aligned}
$$

where

$\mathbf{m}=\int \mathbf{G}^{\mathbf{T}} \rho \mathbf{I} \mathbf{G} \mathrm{d} A$,

$t=$ thickness of the stiffener,

$b=$ breadth of the stiffener.

\subsection{Damping matrix}

In the present work, we use Rayleigh damping in that the damping matrix $\mathbf{C}$ is expressed as

$\mathbf{C}=\alpha \mathbf{K}+\beta \mathbf{M}$,

where $\alpha$ and $\beta$ are the stiffness and mass proportional damping constants.

\subsection{Force vector}

The force vector comprises of two parts; viz. the externally applied time-dependent forces and the timedependent actuator forces generated due to piezoelectric effects. The sensors in the system sense the signal due to externally applied forces. The signal is fed back to the actuator that generates the control forces. In this section, the formulation of the sensor and actuator mechanics is discussed in brief.

\subsubsection{Sensor mechanics}

The equation governing the sensor mechanics is as follows:

$\mathbf{D}^{k}=\mathbf{e}^{k} \boldsymbol{\epsilon}^{k}+\overline{\boldsymbol{\epsilon}}^{k} \mathbf{E}^{k}$,

where $\mathbf{D}^{k}$ corresponds to the electric field displacement vector, $\mathbf{e}^{k}$ is the matrix of piezoelectric constants, $\epsilon^{k}$ is the strain vector, $\overline{\boldsymbol{\epsilon}}^{k}$ is the matrix of dielectric constants, and $\mathbf{E}^{k}$ is the electric field intensity of the $k$ th layer. Integrating the electric field displacement vector over effective area of the electrode surface would give the surface charge collected. In the case of a plate, it is assumed that for a piezoelectric layer acting as a sensor the electric field intensity is zero. Eq. (19) is thus simplified to $D_{3}^{k}=\mathbf{e} \epsilon$.

According to the Gauss law, the closed circuit charge measured through the electrodes of a sensor patch in the $k$ th layer is

$q=\frac{1}{2}\left(\left[\int_{R} D_{3}^{k} \mathrm{~d} A\right]_{z=z_{k}}+\left[\int_{R} D_{3}^{k} \mathrm{~d} A\right]_{z=z_{k-1}}\right)$,

where $R$ is the effective surface electrode of the patch. The effective area is the overlapping area of the electrode surfaces on both sides of the lamina. In case of a sensor the piezoelectric patch acts as the effective electrode surface. Therefore, the above equation transforms to

$q=\int_{R}\left(\mathbf{e B} \boldsymbol{\delta}_{\mathbf{e}}\right) \mathrm{d} A$,

where $\mathbf{e}$ is the matrix of piezoelectric constants, $\mathbf{B}$ is the strain-displacement matrix, and $\boldsymbol{\delta}_{\mathrm{e}}$ is the global displacement vector.

The sensor voltage is computed as follows:

$V_{s}=\frac{q}{C}$,

where

$C=\bar{\varepsilon} \frac{A}{t}=$ capacitance of the piezoelectric layer,

where $\bar{\varepsilon}$ is the permittivity, $A$ is the active (overlap) area of piezoelectric layer, and $t$ is the piezoelectric layer thickness.

\subsubsection{Actuator mechanics}

The converse piezoelectric effect equation is expressed as

$\sigma_{\mathrm{ij}}=\overline{\mathbf{D}} \epsilon_{\mathrm{ij}}-\left(\mathbf{e}^{\mathrm{T}} \mathbf{E}_{\mathrm{j}}\right)$,

where $\overline{\mathbf{D}}$ is the matrix of elastic constants, $\mathbf{E}_{\mathbf{j}}$ is the electric field vector, and $\mathbf{D}_{\mathbf{j}}$ is the electric displacement vector.

The electric field vector is assumed to act across the thickness of the plate so that the electric field intensity is calculated as

$\left(E_{3}\right)_{k}=\frac{V_{k}}{h_{k}}$,

where the subscript 3 refers to the $z$ direction, $h_{k}$ is the thickness of the $k$ th layer, and $V_{k}$ is the voltage across the $k$ th layer. The equivalent force and moments are

$$
\left\{\begin{array}{cc}
N_{x}^{P} & M_{x}^{P} \\
N_{y}^{P} & M_{y}^{P} \\
N_{x y}^{P} & M_{x y}^{P}
\end{array}\right\}=\sum_{k=1}^{\text {Nlay }} \int_{z(k-1)}^{z(k)}\left\{\begin{array}{c}
e_{31} \\
e_{32} \\
0
\end{array}\right\}_{k}(1, z)\left(E_{3}\right)_{k} \mathrm{~d} z .
$$


The nodal force vector is given as

$\mathbf{F}^{\mathbf{P}}=\int \mathbf{B}^{\mathbf{T}}\left\{\begin{array}{c}\mathbf{N}^{\mathbf{P}} \\ \mathbf{M}^{\mathbf{P}}\end{array}\right\} \mathrm{d} A$.

The piezoelectric force effects are also incorporated in the stiffener formulation. The strain-displacement matrix obtained in Eq. (11) is used to compute the nodal forces and moments generated due to piezoelectric layers in the stiffener. Thus,

$\mathbf{F}_{s}^{\mathbf{P}}=\int \mathbf{B}_{s}^{\mathbf{T}}\left\{\begin{array}{l}\mathbf{N}_{s}^{\mathbf{P}} \\ \mathbf{M}_{s}^{\mathbf{P}}\end{array}\right\} \mathrm{d} s$,

where $\mathbf{N}_{\mathrm{s}}^{\mathbf{p}}$ and $\mathbf{M}_{\mathrm{s}}^{\mathbf{p}}$ are the actuator forces and moments generated in the stiffener.

\section{A note on integration}

The integration of Eqs. (12), (17) and (28) is carried out using the Gaussian integration method. In the present formulation the stiffener can have any arbitrary planform (Fig. 2).

Therefore, a special technique is to be adopted to find out the integration points and the Jacobian needed for the transformation. The path of the stiffener within the plate element is defined by three given points. The coordinates of these points in isoparametric co-ordinate system are found out by iterative solution of a biquadratic equation. The Cartesian co-ordinates can be mapped into the isoparametric $(\xi, \eta)$ system by using the shape functions of the plate. The biquadratic equation is given as

$$
\begin{aligned}
& f_{1}=\sum_{r=1}^{n} N_{r} x_{r}-x=0, \\
& f_{2}=\sum_{r=1}^{n} N_{r} y_{r}-y=0,
\end{aligned}
$$

where $N_{r}$ is the shape function for the node $r, n$ is the total number of nodes in the element, and $x_{r}$ and $y_{r}$ are the nodal co-ordinates of the plate. The solution to the set of non-linear equations, i.e., the isoparametric coordinates corresponding to the $(x, y)$ system, can be found out by Newton's iterative technique

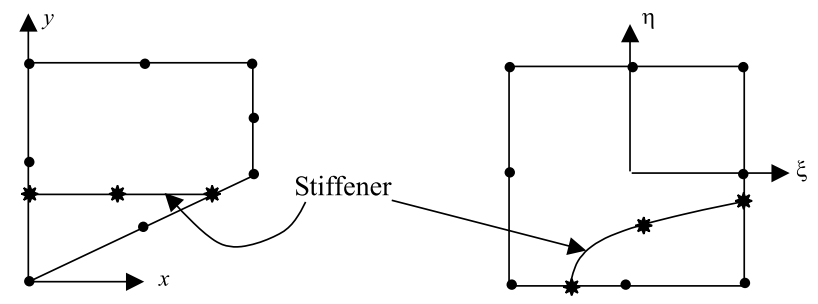

Fig. 2. A stiffener in $x, y$ and $\xi, \eta$ co-ordinates. $\left\{\begin{array}{l}\xi \\ \eta\end{array}\right\}_{i}=\left\{\begin{array}{l}\xi \\ \eta\end{array}\right\}_{i-1}-\mathbf{J}_{i-1}^{-1}\left\{\begin{array}{l}f_{1} \\ f_{2}\end{array}\right\}_{i-1}$,

where

$\mathbf{J}=\left[\begin{array}{ll}\frac{\partial f_{1}}{\partial \xi} & \frac{\partial f_{1}}{\partial \eta} \\ \frac{\partial f_{2}}{\partial \xi} & \frac{\partial f_{2}}{\partial \eta}\end{array}\right]$.

It is to be noted that the planform of the stiffener in the isoparametric co-ordinates need not be a straight line. The general equation of a curve through three points can be expressed as

$\eta=a+b \xi+c \xi^{2}$.

Since the $(\xi, \eta)$ co-ordinates of each of the three points are known, we get a system of three simultaneous equations, which are as follows:

$\left\{\begin{array}{l}\eta_{1} \\ \eta_{2} \\ \eta_{3}\end{array}\right\}=\left[\begin{array}{lll}1 & \xi_{1} & \xi_{1}^{2} \\ 1 & \xi_{2} & \xi_{2}^{2} \\ 1 & \xi_{3} & \xi_{3}^{2}\end{array}\right]\left\{\begin{array}{l}a \\ b \\ c\end{array}\right\}$.

The values of the coefficients $a, b$ and $c$ can be calculated by solving Eq. (32). Since the limits of the $\xi$ coordinates are obtained, they are mapped into another isoparametric $(\alpha)$ system, ranging from -1 to +1 so that the stiffener element becomes a three noded element in $\alpha$ co-ordinate. Therefore,

$\xi=\sum_{i=1}^{3} N_{i}(\alpha) \xi_{i}$

A two-point Gauss integration scheme is followed and the values of $\xi$ corresponding to the Gaussian points in $\alpha$ system are calculated according to Eq. (33). The $\eta$ co-ordinates corresponding to the evaluated values of $\xi$ are found out using Eq. (31). Thus Eq. (11) reduces to

$$
\begin{aligned}
& \mathbf{K}_{s}=\int_{-1}^{1} \mathbf{B}_{s}^{\mathbf{T}} \mathbf{D}_{s} \mathbf{B}_{s} J_{\alpha} \mathrm{d} \alpha, \\
& \therefore \mathbf{K}_{s}=\sum_{i=1}^{\mathrm{NI}} \mathbf{B}_{\mathrm{s}}^{\mathbf{T}} \mathbf{D}_{s} \mathbf{B}_{s} J_{\alpha} \mathrm{d} \alpha w_{i j},
\end{aligned}
$$

where NI is the number of integration points, $w_{i j}$ is the corresponding weight, and $J_{\alpha}$ is the Jacobian for the transformation of co-ordinates. The points of integration are chosen on the $\alpha$ co-ordinate system. Using Eq. (31) these points are mapped on the $(\xi, \eta)$ system and the $(\xi, \eta)$ are mapped to the $x-y$ co-ordinates. The Jacobian $J_{\alpha}$ is written as

$\mathbf{J}_{\alpha}=\frac{\partial x}{\partial \alpha}=\frac{\partial x}{\partial \xi} \frac{\partial \xi}{\partial \alpha}+\frac{\partial x}{\partial \eta} \frac{\partial \eta}{\partial \xi} \frac{\partial \xi}{\partial \alpha}$.

Similarly, the nodal force vector due to actuators bonded to the stiffener is evaluated from Eq. (28) as 
$\mathbf{F}_{s}^{\mathbf{P}}=\sum_{i=1}^{\mathrm{NI}} \mathbf{B}_{s}^{\mathbf{T}}\left\{\begin{array}{c}\mathbf{N}_{s}^{\mathbf{P}} \\ \mathbf{M}_{s}^{\mathbf{P}}\end{array}\right\} J_{\alpha} \mathrm{d} \alpha w_{i j}$

\section{Control algorithm}

Commonly used control algorithms are based on displacement and velocity feedbacks. In the present work a velocity feedback control is employed. The sensor signal is differentiated with respect to time, amplified and fed back to the actuators. Unlike the displacement feedback where along with the formation of control forces the system frequencies also get modified, in velocity feedback the control forces generated by the actuators augment only the damping characteristics of the system. The velocity feedback signal is defined as

$V_{\mathrm{a}}=G \frac{\partial V_{\mathrm{s}}}{\partial t}$,

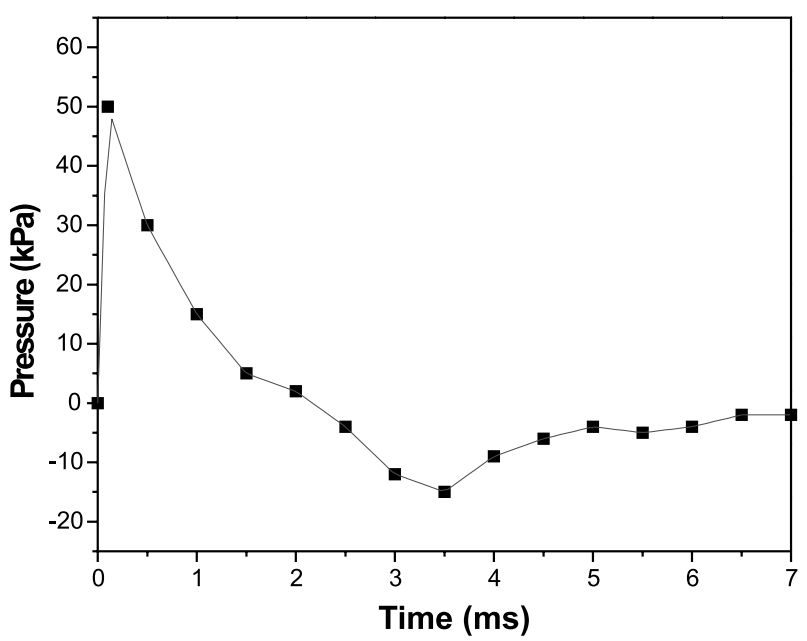

Fig. 3. Air blast [12].

Table 1

Constituent properties of clamped plate

\begin{tabular}{ll}
\hline Thickness, $t$ & $3.4 \mathrm{~mm}$ \\
Young's modulus, $E$ & $207 \mathrm{GPa}$ \\
Poisson's ratio, $v$ & 0.3 \\
Density, $\rho$ & $7770 \mathrm{~kg} / \mathrm{m}^{3}$ \\
Damping ratio, $\xi$ & 0.002 \\
\hline
\end{tabular}

where $V_{\mathrm{a}}$ is the actuator voltage, $G$ is the gain, and $V_{\mathrm{s}}$ is the sensor voltage.

\section{Numerical examples}

In the first example we validate the formulation by comparing our results with the published results. In the subsequent examples, stiffened plates with various boundary conditions are analyzed. In all examples, the piezoelectric layers are treated as self-sensing actuators.

Example 1 (A square clamped plate subjected to air blast). Houlston et al. [12] have experimentally investigated the structural response of a clamped plate under air blast (Fig. 3). The plate $(508 \times 508 \mathrm{~mm})$ is analyzed using a $10 \times 10$ mesh. The constituent properties of the plate are given in Table 1 . The natural frequency parameter $\left(\lambda=\omega a^{2} \sqrt{\rho t / D}\right)$ is compared in Table 2. In this expression, $a$ is the length of the plate and $D$ is the flexural rigidity. The agreement between the natural frequencies predicted by the present technique and that of previous investigators is very good. The displacement time history at the center of the plate is presented with that of the other investigators in Fig. 4.

The plate is analyzed for controlled response using two PZT layers (Table 3), one each at top and bottom. The displacement response is presented in Fig. 5 for different values of gain (Eq. (37)). It is observed that as the gain increases the response attenuates faster. However, the gain is limited a maximum value beyond which instead of attenuation the system response amplifies.

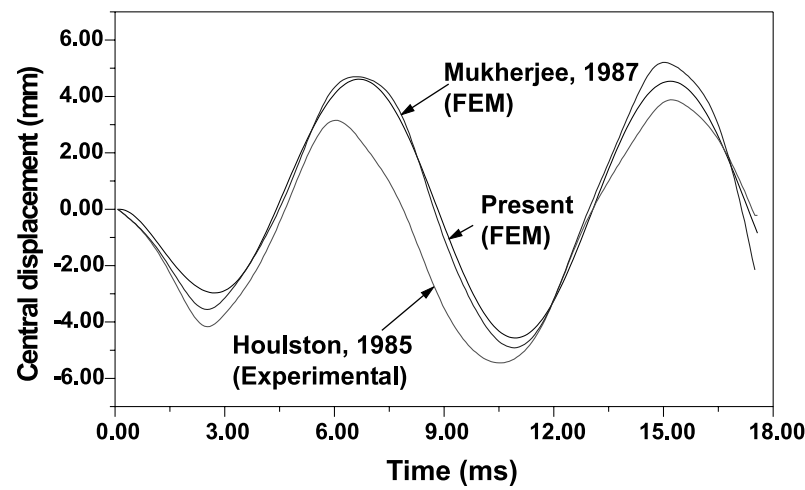

Fig. 4. Displacement response at center of the plate (Example 1).

Table 2

Natural frequency parameter, $\lambda$ (Example 1)

\begin{tabular}{llllll}
\hline & Mode 1 & Mode 2 & Mode 3 & Mode 4 & Mode 5 \\
\hline Mukherjee [9] & 36.00 & 132.66 & 133.49 & 244.28 & 313.43 \\
Present & 35.92 & 130.81 & 131.44 & 217.95 & 305.47 \\
\hline
\end{tabular}


Table 3

Constituent properties of PZT

\begin{tabular}{ll}
\hline Properties & PZT \\
\hline Elastic modulus $\left(E_{11}=E_{22}=E_{33}\right)$ & 63.00 \\
$\quad(\mathrm{GPa})$ & \\
Shear modulus $(\mathrm{GPa})$ & 24.20 \\
Poisson's ratio & 0.30 \\
Density, $\rho\left(\mathrm{N} \mathrm{s} / \mathrm{m}^{4}\right)$ & 7600.00 \\
Thickness, $t(\mathrm{~mm})$ & 0.25 \\
Piezoelectric constants $\left(d_{31}=d_{32}\right)$ & $256 \times 10^{-12}$ \\
$\quad\left(\mathrm{mV}^{-1}\right)$ & \\
Dielectric coefficients $\left(\mathrm{F} \mathrm{m}^{-1}\right)$ & $1.15 \times 10^{-9}$ \\
\hline
\end{tabular}

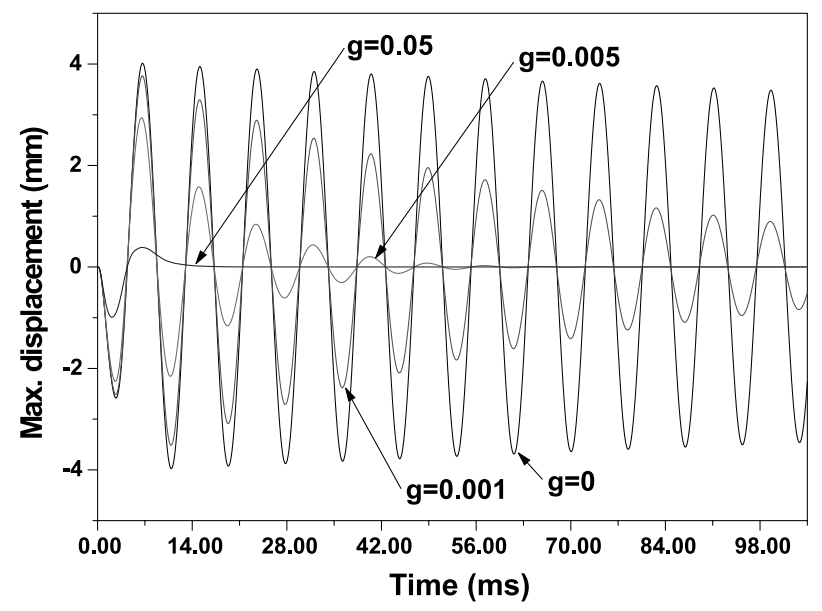

Fig. 5. Displacement response for various gains (Example 1).

Table 4

Material properties of stiffened plate

\begin{tabular}{ll} 
Young's modulus & $7.00 \mathrm{GPa}$ \\
Shear modulus & $2.60 \mathrm{GPa}$ \\
Density & $1504.20 \mathrm{~kg} / \mathrm{m}^{3}$ \\
Poisson's ratio & 0.345 \\
\hline
\end{tabular}

Example 2 (Active vibration control of stiffened plate subjected to air blast). A glass-reinforced polyester plate (Table 4), with two opposite edges free and the other two edges clamped, is analyzed using a $10 \times 10$ finite element mesh. The dimensions of the plate are $300 \mathrm{~mm}$ (free) $\times 375 \mathrm{~mm}$ (clamped). The plate contains a stiffener ( $3 \mathrm{~mm}$ wide and $6 \mathrm{~mm}$ thick) running through the centerline of the plate parallel to the free edges. The thickness of the stiffened plate is $2.32 \mathrm{~mm}$. The material properties are given in Table 3. The natural frequencies of the system corroborate well with the published results $[10,11]$ (Table 5).
The plate is subjected to an air blast (Fig. 3) and the response is controlled by attaching two PVDF layers (Table 6), one each at top and bottom. In the forced vibration analysis first seven modes are considered and the material damping is neglected. The displacement responses for various gains are shown in Fig. 6. The oscillation damps out very quickly as the gain increases. The controlled responses show a shift in the periodicity in comparison to the uncontrolled response. This may be due to the added stiffness of the PVDF layers.

Example 3 (A stiffened cantilever plate subjected to sinusoidal load). A graphite-epoxy (AS4/3501) crossply laminated $(0 / 90)$ s cantilever plate $(508 \mathrm{~mm} \times$ $508 \mathrm{~mm} \times 2 \mathrm{~mm}$ thick) is subjected to a sinusoidal load $\left(f=f_{0} \sin (5 \pi t)\right)$ for a duration of $0.5 \mathrm{~s}$. The responses of the structure are observed for a duration of $3 \mathrm{~s}$. The plate is stiffened with two stiffeners $(5 \mathrm{~mm}$ wide and 10 $\mathrm{mm}$ thick). The stiffeners are placed at a 3/5th distance from the centerline along the width of the plate. The material properties of the plate and the stiffeners are given in Table 7. Two PZT layers (Table 2), each attached at top and bottom of the plate, are used to control the vibration. Taking advantage of symmetry about $x$-axis, only one half structure is modeled using a $10 \times 5$ finite element mesh (Fig. 7). The normalized tip displacement response for different gains is presented in Fig. 8. It is seen that the attenuation is predominant in the free vibration phase. The enlarged view of the response is shown in Fig. 9 to compare the attenuation rate for different gains.

The same plate is analyzed without the stiffeners and the normalized tip displacement responses are obtained (Fig. 10). The results show a marked difference in the response pattern between the stiffened and bare plates. In case of the bare plate, as the gain is increased the response shows participation of higher modes. Thus, it can be said in this case that the actuation tends to excite higher modes.

Example 4 (Cantilever plate with stiffener not passing through the nodal line). The present stiffener formulation allows the stiffener to be independent of the finite element mesh. The efficacy of this formulation is examined in this example. The plate in Example 3 is reanalyzed by changing the mesh division so that the stiffener passes

Table 5

Natural frequencies of stiffened plate $(\mathrm{Hz})$

\begin{tabular}{lllllll}
\hline & Mode 1 & Mode 2 & Mode 3 & Mode 4 & Mode 5 & Mode 6 \\
\hline Theoretical [14] & 68.61 & 71.20 & 124.70 & 150.40 & 183.20 & 184.80 \\
Mukherjee [15] & 68.31 & 69.07 & 118.40 & 160.60 & 179.10 & 179.70 \\
Present & 68.47 & 68.66 & 119.59 & 162.16 & 177.11 & 177.39 \\
\hline
\end{tabular}


Table 6

Properties of PVDF

\begin{tabular}{ll}
\hline Properties & PVDF \\
\hline Elastic modulus $\left(E_{11}=E_{22}=E_{33}\right)(\mathrm{GPa})$ & 2.00 \\
Shear modulus $(\mathrm{GPa})$ & 0.776 \\
Poisson's ratio & 0.30 \\
Density, $\rho\left(\mathrm{kg} / \mathrm{m}^{3}\right)$ & 1800 \\
Thickness, $t(\mathrm{~mm})$ & 0.50 \\
Piezoelectric constants $\left(e_{31}=e_{32}\right)$ & 0.046 \\
$\quad\left(\mathrm{mV}^{-1}\right)$ & \\
Dielectric coefficients $\left(\mathrm{F} \mathrm{m}^{-1}\right)$ & $1.062 \times 10^{-10}$ \\
\hline
\end{tabular}

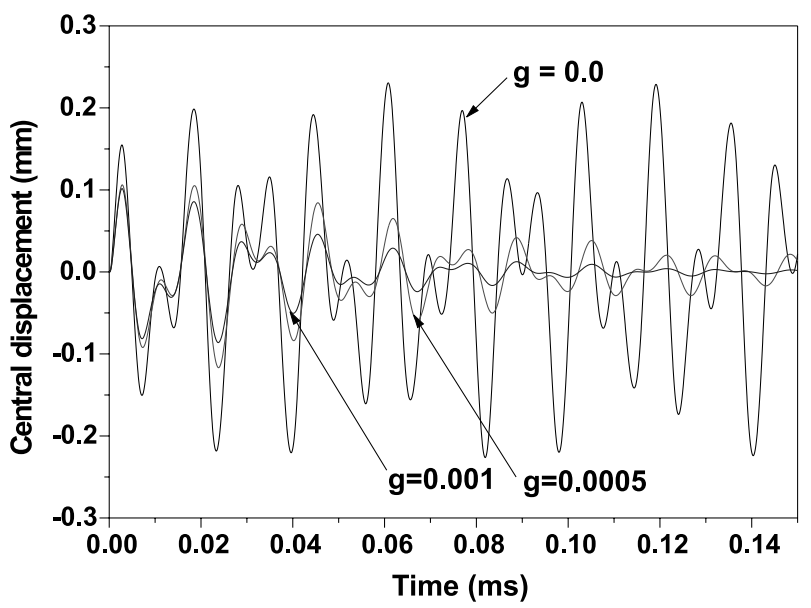

Fig. 6. Displacement response for various gains (Example 2).

Table 7

Properties of AS4/3501 graphite-epoxy plate

\begin{tabular}{ll}
\hline Properties & AS4/3501 \\
\hline Elastic modulus $\left(E_{11}=E_{33}\right), E_{22}(\mathrm{GPa})$ & $144.80,9.67$ \\
Shear modulus $\left(G_{12}=G_{13}\right), G_{23}(\mathrm{GPa})$ & $4.14,3.45$ \\
Poisson's ratio & 0.30 \\
Density, $\rho\left(\mathrm{N} \mathrm{s}{ }^{2} / \mathrm{m}^{4}\right)$ & 1389.00 \\
Damping ratio, $\xi$ & 0.01 \\
\hline
\end{tabular}

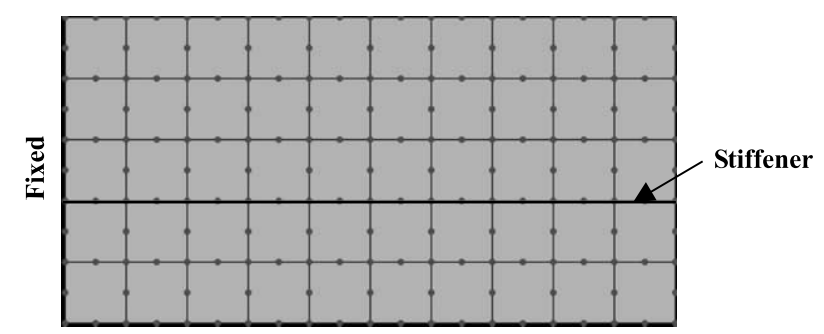

Fig. 7. Cantilever plate with stiffener passing through the nodes.

through the lower one-third portion of the row of the elements (Fig. 11). The location of the stiffener on the plate is same as in the previous example. The gain in this case is 0.001 . The natural frequencies of the system compare well with those in Example 3 (Table 8). The

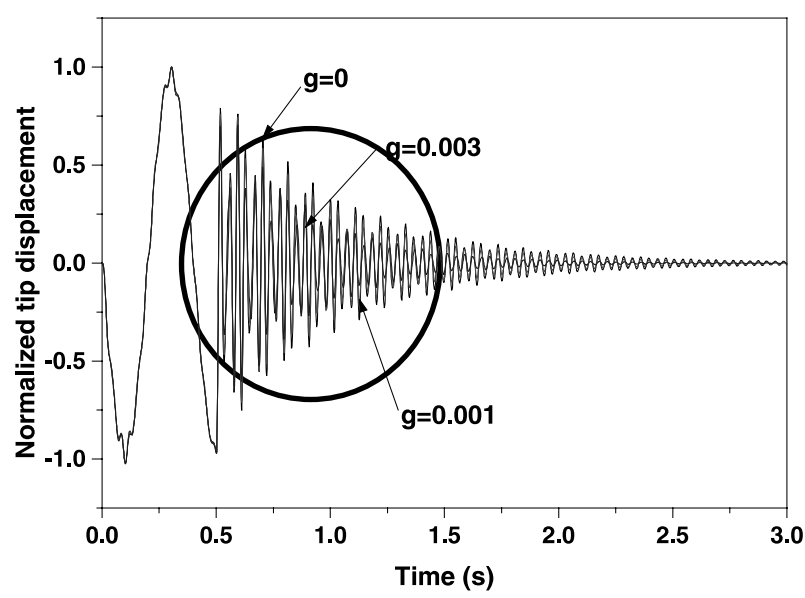

Fig. 8. Normalized tip displacement response of the stiffened plate for various gains (Example 3).

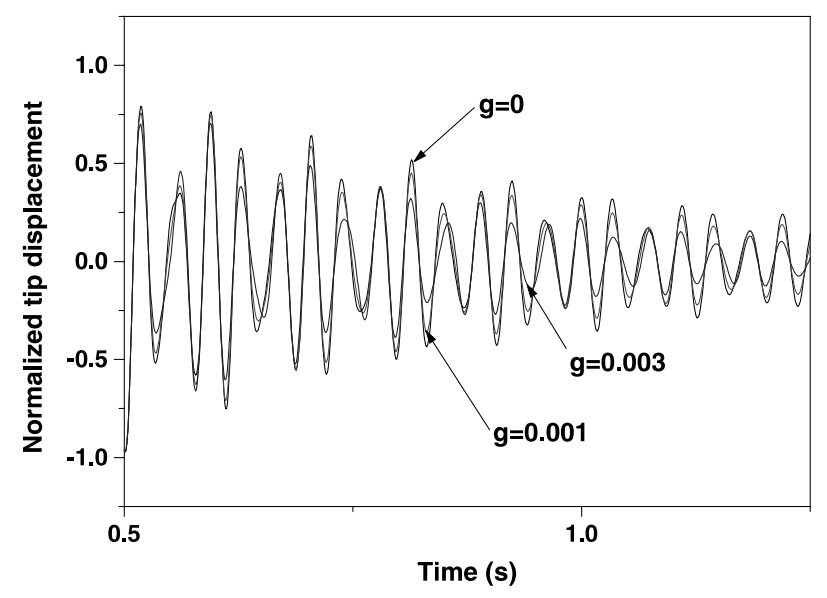

Fig. 9. Enlarged view of the normalized tip displacement response (Example 3).

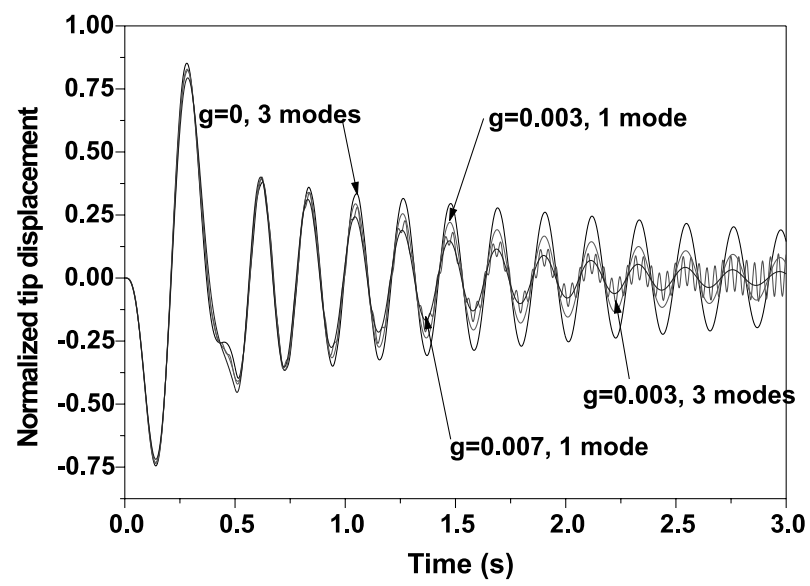

Fig. 10. Normalized tip displacement response of the bare plate for various gains (Example 3).

displacement response in both the cases compares well (Fig. 12). This validates the formulation when the stiffener is away from the nodal lines. 


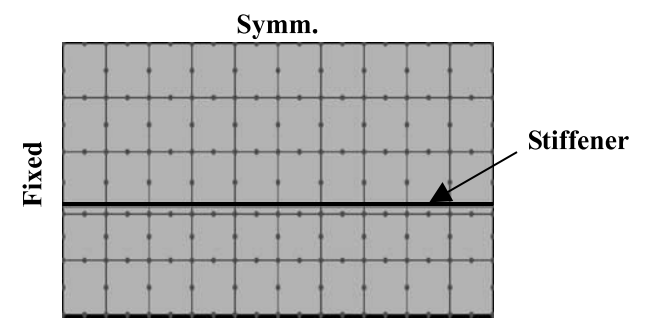

Fig. 11. Cantilever plate with stiffener away from the nodal line (Example 4).

Table 8

Natural frequencies $(\mathrm{Hz})$ of the stiffened plate (Example 4)

\begin{tabular}{llll}
\hline & Mode 1 & Mode 2 & Mode 3 \\
\hline Example 3 & 27.25 & 37.00 & 72.52 \\
Example 4 & 27.50 & 37.22 & 74.46 \\
\hline
\end{tabular}

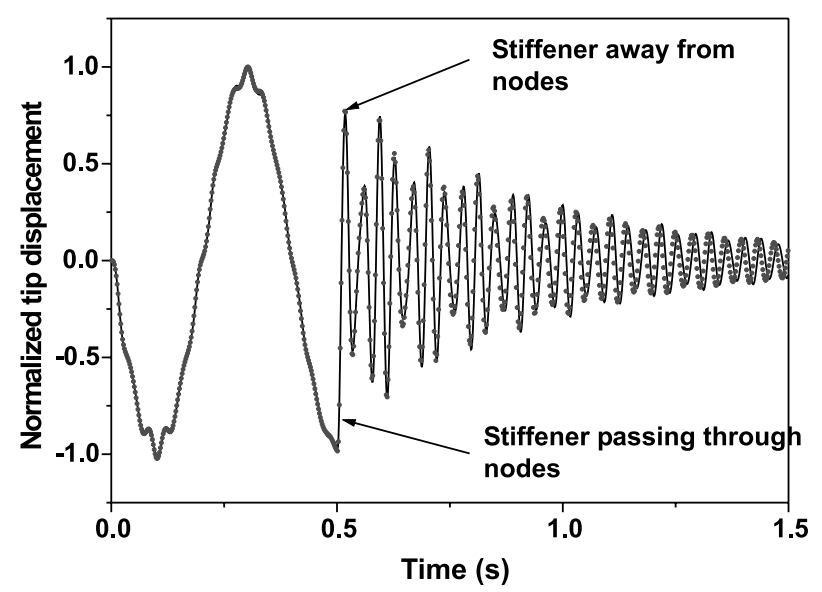

Fig. 12. Normalized tip displacement response with stiffener passing through nodal line and with stiffener placed away from the nodal line $(g=0.001)$ (Example 4).

\section{Closing remarks}

In this paper, the active control of stiffened composite plates using piezoelectric materials has been presented. The stiffener has been formulated in such a way that it can have any shape in plan and need not pass through the nodal lines of the finite element mesh. The efficacy of the formulation with the stiffeners away from nodal lines has been established. This feature is helpful in analyzing the plates with irregular shapes. Several problems of stiffened plates under a variety of excitations have been solved. The effect of altering gain in actuation has been studied. It is observed from one of the examples that the actuation effect tends to excite higher-order modes. This needs to be verified by solving similar problems. Further, the present formulation can be effectively employed in the analysis of plates with electrical stiffeners. The electrical stiffeners are the stiffeners with piezoelectric effects and may prove effective in vibration control as by changing the applied voltage the stiffening effect could be altered. Results of this investigation will be reported shortly.

\section{References}

[1] Bailey T, Hubbard JE. Distributed piezoelectric-polymer active vibration control of a cantilever beam. AIAA J Guidance and Control 1985;8:605-11.

[2] Anderson EH, Hagood NW. Simultaneous piezoelectric sensing/ actuation: analysis and application to controlled structures. J Sound Vib 1994;174:617-39.

[3] Tzou HS. Piezoelectric Shells-Distributed Sensing and Control of Continua. Dordrecht: Kluwer Academic Publishers; 1993.

[4] Gaudenzi P, Carbonaro R, Benzi E. Control of beam vibrations by means of piezoelectric devices: theory and experiments. J Compos Struct 2000;50:373-9.

[5] Lam KY, Ng TY. Active control of composite plates with integrated piezoelectric sensors and actuators under various dynamic loading conditions. Smart Mater Struct 1999;8:223-37.

[6] Chandrashekhara K, Agarwal AN. Active vibration control of laminated composite plates using piezoelectric devices: a finite element approach. J Intell Mater Syst Struct 1993;4:496-508.

[7] Birman V, Adali S. Vibration damping using piezoelectric stiffener-actuators with application to orthotropic plates. Compos Struct 1996;35:251-61.

[8] Birman V. Optimum distribution of smart stiffeners in sandwich plates subjected to bending or forced vibrations. Composites: Part B 1996;27B:657-65.

[9] Young AJ, Hansen CH. Control of flexural vibrations in stiffened structures using multiple piezoelectric actuators. Appl Acoustics 1996;49:17-48.

[10] Goswami S, Kant T. Active vibration control of intelligent stiffened laminates using smart piezoelectric materials by finite element method. J Reinf Plastics Compos 1998;16:1472-93.

[11] Mukherjee A, Joshi SP. Design of actuator profiles for minimum power consumption. Smart Mater Struct 2001;10:305-13.

[12] Houlston R, Slater JE, Pegg N, Des Rochers GG. On analysis of structural response of ship panels subjected to air blast loading. Compos Struct 1985;21:273-89.

[13] Mukherjee A. On finite element dynamic and stability analyses of stiffened plate structures. PhD Thesis, Department of Naval Architecture, IIT Kharagpur, 1987.

[14] Attaf B, Hollaway L. Vibrational analyses of stiffened and unstiffened composite plates subjected to in-plane loads. Composites 1990;21:117-26.

[15] Mukherjee A. Free vibration of laminated beams and stiffened plates using a high-order element. Aeronautical J 1991;95:124-31. 